\title{
Comparison of digital subtraction angiography and contrast-enhanced ultrasound in assessment of carotid stenosis
}

\author{
Ronggui Liu, Zhimei Yan, Guijun Zhang, Zhaoyan Ding, Yunping Li, Zhirong Jiang
}

Department of ultrasound, The Affiliated Hospital of Qingdao University, Qingdao, Shandong Province, China, 266555.

\begin{abstract}
Introduction: The performances of contrast-enhanced ultrasound (CEUS) and digital subtraction angiography (DSA) were used to establish an efficient as well as non-invasive clinical technique for the diagnosis of extra-cranial internal carotid artery (ICA) stenosis.

Materials and methods: Thirty-six successive patients (11 women and 25 men, mean age: $65.0 \pm 9.2$, range: 43-78 years) with internal carotid artery (ICA) stenosis were tested by CEUS and DSA. These tests were carried out by means of Hitachi Preirus ultrasound machine for CEUS and Allura Xper FD20 system (Philips Medical Systems, Nederland B.V.) for DSA. $1.2 \mathrm{ml}$ SonoVue (Bracco, Switzerland) was used a s contrast agent.

Results: The results clearly indicated that there were no noteworthy variations among the distributions recorded by CEUS as well as DSA for the four tested groups. The percentage of diameter stenosis calculated by CEUS was clearly in accordance with the DSA images. CEUS showed accurate results with good specificity and sensitivity at $50 \%, 70 \%$, and $100 \%$. Also, CEUS performance was relatively better than DSA in the diagnosis of ICA and suitability of CEA.

Conclusion: CEUS proved to be a precise non-invasive testing method for the diagnosis of carotid artery stenosis which is more feasible and well-tolerated in patients with various stages of carotid stenosis.

Keywords: Carotid artery stenosis, CEUS, contrast-enhanced ultrasound, digital subtraction angiography.

DOI: https://dx.doi.org/10.4314/ahs.v20i1.57

Cite as: Liu R, Yan Z, Zhang G, Ding Z, Li Y, Jiang Z. Comparison of digital subtraction angiography and contrast-enhanced ultrasound in assessment of carotid stenosis. Afri Health Sci. 2020;20(1):509-14. https:/ / dx.doi.org/ 10.4314/ abs.v20i1.57
\end{abstract}

\section{Introduction}

Carotid endarterectomy (CEA) or carotid artery stenting (CAS) is an operative method carried out to adjust stenosis (narrowing) in the internal carotid artery (ICA) ${ }^{1}$. The suitability of the patient to this procedure was tested on the basis of digital subtraction angiography (DSA). Consequently, the patients having an ipsilateral carotid
Corresponding author:
Zhirong Jiang,
Department of ultrasound,
The Affiliated Hospital of
Qingdao University, No. 1677
Wutai mountain road, Huangdao District,
Qingdao, Shandong Province, 266555,
Email: zjiang11@yahoo.com

occlusion were regarded inappropriate for this surgery. Therefore, it is a critical task as DSA is the only reference standard to assess the carotid stenosis but being an invasive procedure, it is accompanied with a minor risk of post-agiography stroke $\mathrm{e}^{2-3}$ On the other hand, ultrasonography being a non-invasive procedure, affords informative indications required for the diagnosis of carotid artery stenosis.

In addition, contrast-enhanced ultrasound (CEUS) imaging has been explored and progressively appeared in clinical technology over the past few decades. Furthermore, contemporary with the technological enhancements in the ultrasound scanning equipments, a wide range of other devices have been intended to encounter the requirements of this quickly growing domain of imaging. However, the rapid development of ultrasound technology is hastened by the functioning issues of the 
doppler procedures and ultrasound imaging. Ultrasound is employed in the investigation of sub-terranean body structures, while the spatial resolution of grayscale imaging and doppler sensitivity get affected with the impactful extent over clinical efficacy of ultrasound ${ }^{4}$. In addition, contrast agents assure the enhancement of specificity and sensitivity of present ultrasound diagnoses as well as possess the potential to extend wide scope of ultrasound applications. This innovative technique, CEUS is potentially used in the quantification of extracranial ICA stenosis in symptomatic patients with ipsilateral ICA apparent occlusion on conventional US.

This study is significantly anticipated to compare CEUS with the standard reference DSA in the patients with apparent carotid stenosis by making use of conventional ultrasound as well as has explored the clinical expediency of CEUS intended for patients suffering from carotid artery stenosis.

\section{Materials and methods}

All the patients were provided with printed informed consent.

\section{Patients}

This study was a retrospection from April 2012 until March 2014 of 68 carotid artery samples taken from 36 patients (11 women and 25 men, mean age: $65.0 \pm 9.2$, range: $43-78$ years) were evaluated. All the patients were investigated with color doppler flow imaging, B-mode ultrasound, CEUS, and DSA.

\section{Equipment}

All the ultrasound examinations were performed by the radiologist with about 5 years experience in vascular ultrasound. CEUS and Conventional ultrasound tests were executed by means of a Hitachi VISION Preirus System (Hitachi Medical Corporation, Tokyo, Japan) with 5-13 $\mathrm{MHz}$ broadband linear array probes. All the examinations were concluded within 2 weeks by means of CEUS, conventional ultrasound, and DSA. Followed by ultrasound procedure, the patients were further investigated by DSA.

\section{Conventional ultrasound}

Conventional ultrasound tests were performed for all the patients along with gray-scale as well as color doppler ultrasound imaging. Peak systolic velocity (PSV) values of the distal extra-cranial ICA were recorded. Supplementary PSV indices were analyzed.

\section{CEUS}

Hitachi HI VISION Preirus machine (Hitachi Medical Corporation, Tokyo, Japan) was utilized with the (contrast) software integrated in the Hitachi system. Contrast-enhanced imaging bearing low-mechanical-index cadence contrast pulse sequencing technology SonoVue (Bracco, Geneva, Switzerland) was employed.

\section{DSA}

Allura Xper FD20system (Philips Medical Systems, Nederland B.V.) was used to carry out intra-arterial DSA within 15 days of the sonographic tests. However, obstructive ICA stenosis were categorized as $0-50 \%$ (mild), 50-69\% (moderate), $70-99 \%$ (severe), and 100\% (occlusion).

\section{Statistics}

Software Statistical Product and Service Solutions 17.0 (Chicago, IL, USA) were used to perform statistical analysis. The mean \pm standard deviation is used to express distribution values while median (M) and inter-quartile range (IQR) is considered to express non-normal distribution values.

\section{Results}

In the course of experimental study, 36 patients were selected for CEUS and DSA. No contrast agents used have instigated undesirable effects. Sixty-eight carotid arteries (11 women and 25 men, mean age: $65.0 \pm 9.2$, range: $43-$ 78 years) were evaluated.

Comparison of CEUS and DSA in the detection of carotid artery stenosis

Distribution of the four groups detected by DSA and CEUS were tabulated in Table-1. No noteworthy variation in the distribution values were so far noticed both by DSA and CEUS for all the four groups $(p=0.01)$. In addition, the percent diameter stenosis values calculated by CEUS imaging intensely correlated with the DSA measurements $(\mathrm{r}=0.97, \mathrm{p}=0.01)$. 


\section{Table-1: Contrast between the performance results of CEUS and digital subtraction angiography}

\begin{tabular}{|c|c|c|c|c|c|}
\hline \multirow{2}{*}{$\begin{array}{c}\text { Total numbers in the four degrees of } \\
\text { carotid artery stenosis groups }\end{array}$} & \multicolumn{5}{|c|}{ CEUS } \\
\cline { 3 - 7 } & $\begin{array}{c}0- \\
49 \%\end{array}$ & $\begin{array}{c}50- \\
69 \%\end{array}$ & $70-99 \%$ & $100 \%$ \\
\hline \multirow{3}{*}{ DSA } & $0-49 \%$ & 23 & 0 & 0 & 0 \\
\cline { 2 - 7 } & $50-69 \%$ & 1 & 5 & 4 & 0 \\
\cline { 2 - 6 } & $70-99 \%$ & 0 & 2 & 24 & 0 \\
\cline { 2 - 6 } & $100 \%$ & 0 & 0 & 1 & 6 \\
\hline
\end{tabular}

Depending upon the four stages of carotid artery stenosis, $50 \%, 70 \%$, and $100 \%$ were taken as standard values to record the specificity and sensitivity DSA and CEUS for evaluating the extent of carotid artery stenosis while the DSA findings were presumed to represent the actual condition. CEUS recordings were in accordance for 7 of 8 vessels (86\% sensitivity) for total occlusion, 31 of 33 vessels (92\% sensitivity) for $\geq 70 \%$ stenosis and 41 of 42 vessels (96\% sensitivity) for $\geq 30 \%$ stenosis (as shown in Table-2).

Table-2: The sensitivity, specificity, positive, and negative predicted values for CEUS at various cut-off points with the DSA results being the reference standard for actual condition. Note: +ve refers to positive; -ve refers to negative.

\begin{tabular}{|l|l|l|l|l|}
\hline $\begin{array}{l}\text { Degree of carotid } \\
\text { artery stenosis } \\
\text { observed by CEUS }\end{array}$ & sensitivity & specificity & $\begin{array}{l}+ \text { ve } \\
\text { predicted } \\
\text { value (\%) }\end{array}$ & $\begin{array}{l}\text {-ve } \\
\text { predicted } \\
\text { value (\%) }\end{array}$ \\
\hline For occlusion & 86 & 100 & 100 & 98 \\
\hline For $70-100 \%$ & 92 & 90 & 91 & 94 \\
\hline For $50-100 \%$ & 96 & 99 & 100 & 96 \\
\hline
\end{tabular}

CEUS versus DSA in the patency estimation of ICA along with suitability for CEA

From the patency assessment findings (shown in Table-3 and Table-4) by CEUS versus DSA, the DSA was diagnosed as carotid artery occlusion for one patient while the CEUS evaluation of the same patient was also carotid artery stenosis $>90 \%$ (Shown in Figure-1). 
Table-3: Performance of CEUS versus DSA in evaluating patency of the ICA

\begin{tabular}{|c|c|c|c|}
\hline \multirow{2}{*}{\multicolumn{2}{|c|}{ Number of carotid arteries }} & \multicolumn{2}{|c|}{ CEUS } \\
\hline & & patent & occluded \\
\hline \multirow{2}{*}{ DSA } & patent & 0 & 1 \\
\hline & occluded & 1 & 11 \\
\hline
\end{tabular}

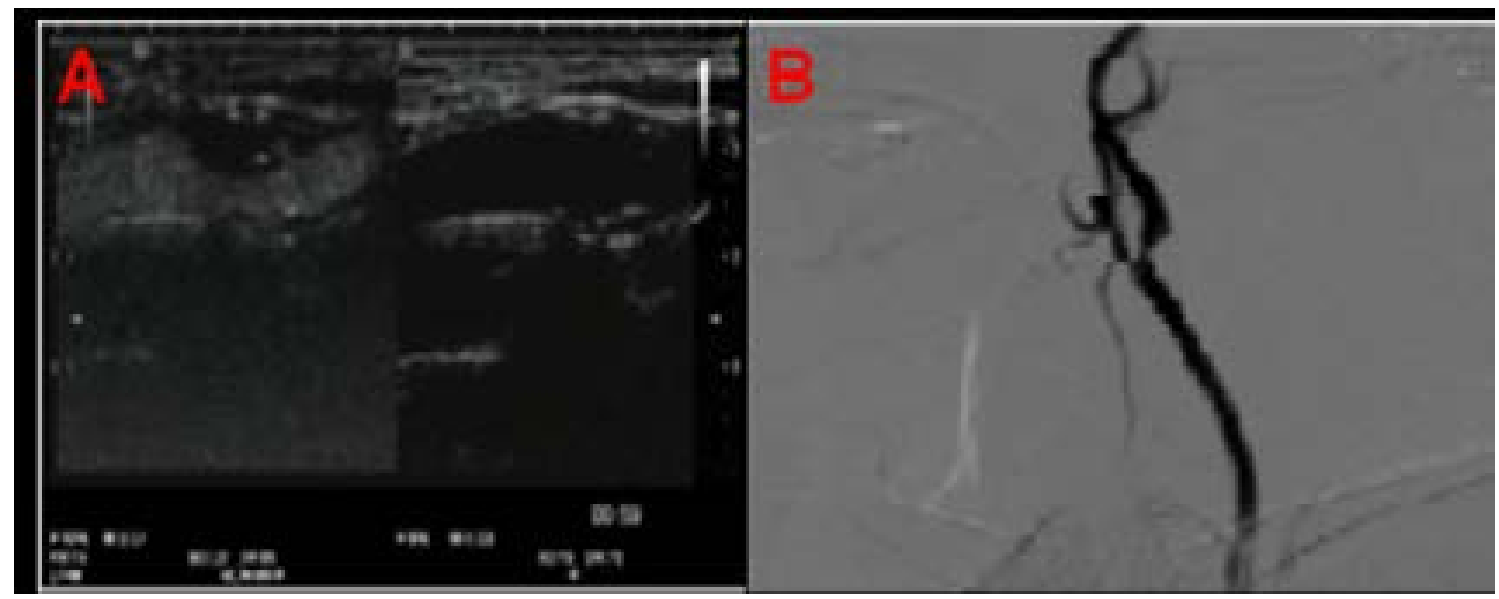

Figure-1: CEUS analysis of a patient with ICA stenosis. (A) An image displaying luminal stenosis and plaque ulceration (arrow) followed by the use of contrast agent. (B) Lateral DSA images of the corresponding patient.

Table-4: Comparison between CEUS and DSA in the assessment of CEA suitability

\begin{tabular}{|c|c|c|c|}
\hline \multicolumn{2}{|c|}{ Number of carotid arteries } & \multicolumn{2}{c|}{ CEUS } \\
\cline { 3 - 4 } & suitable & unsuitable \\
\hline \multirow{2}{*}{$\begin{array}{c}\text { DSA (suitability for } \\
\text { CEA) }\end{array}$} & suitable & 0 & 1 \\
\cline { 2 - 4 } & unsuitable & 1 & 11 \\
\hline
\end{tabular}

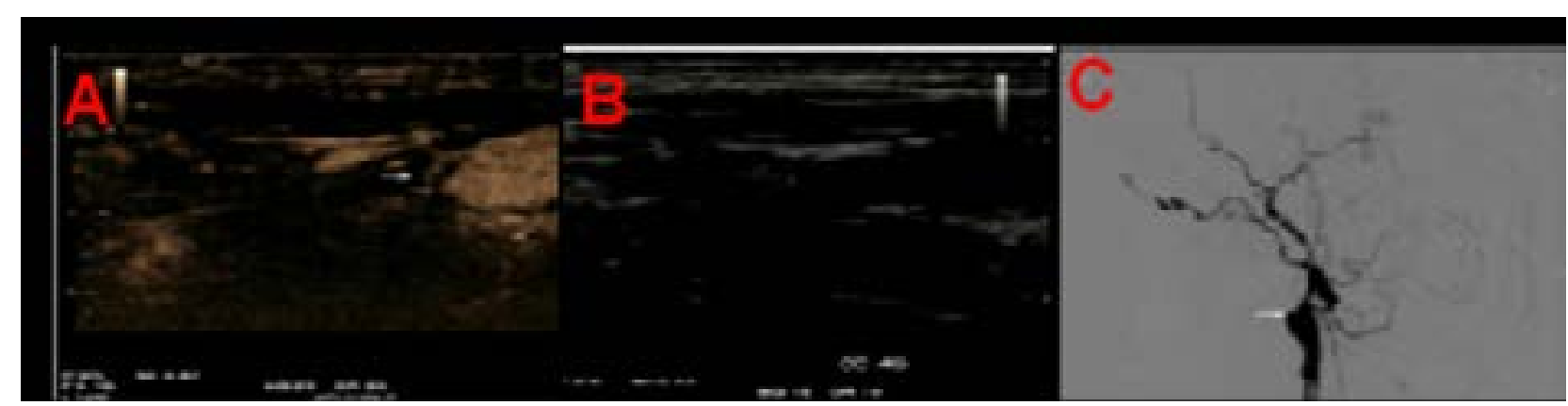

Fig.2. (A) An image displaying slender flow (arrow) after the use of (SonoVue) a contrast agent. (B) An image displaying slender flow (arrow) before the use of SonoVue. (C) DSA images of the corresponding patient displaying a small stump of the proximal ICA (arrow) with no noticeable flow away from it, representing a proximal ICA occlusion. 


\section{Discussion}

Severe carotid artery stenosis diagnosed patients are subjected to extreme chances of stroke. Increasing obstruction of the ICA apparently increases these chances of ischemic stroke while the degree of stenosis being one of the significant factors to decide the patient selectivity for surgical CEA ${ }^{8}$. DSA is considered as a golden standard in the diagnosis of vascular diseases, however possess several complications. CEUS is a latest technological innovation that has the potential to perform microvascular imaging ${ }^{7,8}$. However, numerous benefits of CEUS turn it to be a more effective technology in contrast to magnetic resonance imaging (MRI), computed tomography (CT) and digital subtraction angiography (DSA).

Microbubble contrast agents along with various other acquisition techniques are available to considerably improve signals from the blood displaying the vascular lumen. Especially, SonoVue is a chemical substance that is injected in to the blood pool for clearer visibility of the ultrasound pictures, which is stabilized by a phospholipid core filled with sulfur hexafluoride gas9. In the present study, we have monitored stenosis as well as carotid plaques inside the carotid artery repeatedly. There were no side effects identified instantaneously or even after 24-hour follow-up duration. Images of carotid stenosis and carotid plaque identified in the CEUS and DSA images respectively were represented in Figure 1. In carotid imaging, sonographic contrast agents were employed to increase signals in the blood stream as well as to increase delineation of vessel walls facilitating the investigation of carotid plagues and intima media thickness (IMT) ${ }^{10}$. From Fig.2, it was clear that DSA diagnosed as occlusion of the internal carotid artery while CEUS also diagnosed the presence of slight occlusion in the bloodstream. Prospective comparison of contrast-enhanced ultrasound and digital subtraction angiography was brought about by the ultrasound imaging modalities.

In this experiment, we have employed CEUS and DSA to diagnose stenosis in 68 ICA and compared these methods against conventional DSA as a standard reference. A better specificity and sensitivity was observed for these two techniques at the values of $50 \%, 70 \%$, and $100 \%$. As per our observation, the possible reason for CEUS results showing stenosis rate lower than that of DSA in one patient might be the presence of acute stenosis portion in the distal internal carotid artery. Consequently, extended research would be desirable to assess the promising clinical expediency of contrast enhanced ultrasound imaging (CEUS) in the determination and proper diagnosis of ICA stenosis of carotid artery.

\section{Conclusion}

CEUS has the ability to enhance better visibility of the blood vessels thus providing a precise interpretation of plaques, ulcers, and carotid stenosis. CEUS is a precise noninvasive imaging technique for the diagnosis of carotid artery stenosis which is feasible as well as highly tolerable in patients with various extents of carotid stenosis.

\section{Conflict of interest}

None declared.

\section{References}

1. Augoustides JG. Advances in the Management of Carotid Artery Disease: Focus on Recent Evidence and Guidelines. J Cardiothorac Vasc Anesth. 2012; 26:16671.

2. Incalzi RA, Gemma A, Landi F, Carbonin PU. Randomised trial of endarterectomy for recently symptomatic carotid stenosis: final results of the MRC European Carotid Surgery Trial (ECST). Lancet 1998; 9113: 1379-87.

3. Leffers AM, Wagner A. Neurologic complications of cerebral angiography-A retrospective study of complication rate and patient risk factors. Acta Radiol. 2000; 41:204-10.

4. Mansour MA, Mattos MA, Hood DB, Hodgson KJ, Barkmeier LD, Ramsey DE. Detection of total occlusion, string sign, and pre-occlusive stenosis of the internal carotid artery by color-flow duplex scanning. Am J Surg. 1995; 170:154-158.

5. Hohmann J, Albrecht T, Oldenburg A, et al. Liver metastases in cancer: detection with contrast-enhanced ultrasonography. Abdom Imaging. 2004; 29:669-81.

6. Hobson2nd RW, Mackey WC, Ascher E, Murad $\mathrm{MH}$, Calligaro KD, Comerota AJ. Management of atherosclerotic carotid artery disease: clinical practice guidelines of the Society for Vascular Surgery. J Vasc Surg. 2008; 2: 480-6.

7. Luo Y, Fan YT, Lu Q, Li B, Wen TF, Zhang ZW. CEUS: A new imaging approach for postoperative vascular complications after right-lobe LDLT. World J Gastroenterol. 2009; 15:3670-3675. doi: 10.3748/wjg.15.3670. 
8. Rennert J, Dornia C, Georgieva M, Roehrl S, Broillet A, Ventrone R, Yan F. BR1: a new ultra sonoFellner C, Schleder S, Stroszczynski C, Jung EM. Identi- graphic contrast agent based on sulfur hexafluoride-filled fication of early complications following liver transplantation using contrast enhanced ultrasound (CEUS). First results. J Gastrointestin Liver Dis. 2012; 21: 407-412. 9. Schneider M, Arditi M, Barrau MB, Brochot J, microbubbles. Invest Radiol. 1995; 30: 451-7.

10. Kono Y, Pinnell SP, Sirlin CB, Sparks SR, Georgy B, Wong W, Mattrey RF. Carotid arteries: contrast enhanced US angiography-preliminary clinical experience. Radiology. 2004; 230: 561-68. 